\title{
A NOTE ON BRIDGELAND'S HALL ALGEBRA OF TWO-PERIODIC COMPLEXES
}

\author{
SHINTAROU YANAGIDA
}

Abstract. We show that the Hall algebra of two-periodic complexes, which is recently introduced by T. Bridgeland, coincides with the Drinfeld double of the ordinary Hall bialgebra.

\section{INTRODUCTION}

0.1. The main object of this paper is the Hall algebra of $\mathbb{Z}_{2}(:=\mathbb{Z} / 2 \mathbb{Z})$-graded complexes, which was introduced by Bridgeland [1].

Let $\mathcal{A}$ be an abelian category over a finite field $\mathfrak{k}:=\mathbb{F}_{q}$ with finite dimensional morphism spaces. Let $\mathcal{P} \subset \mathcal{A}$ be the subcategory of projective objects. Let $\mathcal{C}(\mathcal{A}) \equiv \mathcal{C}_{\mathbb{Z}_{2}}(\mathcal{A})$ be the abelian category of $\mathbb{Z}_{2}$-graded complexes in $\mathcal{A}$. An object of $\mathcal{C}(\mathcal{A})$ is of the form

$$
M_{1} \underset{g}{\stackrel{f}{\rightleftarrows}} M_{2}, \quad f \circ g=0, g \circ f=0 .
$$

Let $\mathcal{C}(\mathcal{P})$ be the subcategory of complexes consisting of projectives, and $\mathcal{H}(\mathcal{C}(\mathcal{P}))$ be its Hall algebra. One can introduce the twisted Hall algebra $\mathcal{H}_{\text {tw }}(\mathcal{C}(\mathcal{P})$ ) as the twisting of $\mathcal{H}(\mathcal{C}(\mathcal{P}))$ by the Euler form of $\mathcal{A}$. In 11, Bridgeland introduced an algebra $\mathcal{D H}(\mathcal{A})$, which is the localization of the twisted Hall algebra $\mathcal{H}_{\mathrm{tw}}(\mathcal{C}(\mathcal{P}))$ by the set of acyclic complexes:

$$
\mathcal{D H}(\mathcal{A}):=\mathcal{H}_{\mathrm{tw}}(\mathcal{C}(\mathcal{P}))\left[\left[M_{\bullet}\right]^{-1} \mid H_{*}\left(M_{\bullet}\right)=0\right]
$$

The purpose of this note is to show the following theorem, which was stated in [1, Theorem 1.2].

Theorem. Assume that the abelian category $\mathcal{A}$ satisfies the conditions

- essentially small with finite morphism spaces,

- linear over $\mathfrak{k}$,

- of finite global dimension and having enough projectives,

- hereditary,

- nonzero object defines nonzero class in the Grothendieck group.

Then the algebra $\mathcal{D H}(\mathcal{A})$ is isomorphic to the Drinfeld double of the bialgebra $\widetilde{\mathcal{H}}(\mathcal{A})$ as an associative algebra.

Here $\widetilde{\mathcal{H}}(\mathcal{A})$ is the (ordinary) extended Hall bialgebra, which will be recalled in $\$ 1.1 .1-1.1 .4$ For the review of the Drinfeld double, see $₫ 1.1 .5$. The proof will be explained in $₫ 2$

The organization of this note is as follows. In $\S 1$, we review Bridgeland's theory [1] and prepare notations and statements which are necessary for the proof of the main theorem. In the subsection \$1.1 we recall the ordinary theory of Hall algebra introduced by Ringel [10. The next subsection $\$ 1.2$ is devoted to the recollection of Bridgeland's theory.

The section $\$ 2$ is devoted to the proof of the main theorem.

We close this note by mentioning some consequences of the theorem in $\$ 3$,

0.2 . Notations and conventions. We indicate several global notations.

$\mathfrak{k}:=\mathbb{F}_{q}$ is a fixed finite field unless otherwise stated, and all the categories will be $\mathfrak{k}$-linear. We choose and fix a square root $t:=\sqrt{q}$.

For an abelian category $\mathcal{A}$, we denote by $\operatorname{Obj}(\mathcal{A})$ the class of objects of $\mathcal{A}$. For an object $M$ of $\mathcal{A}$, the class of $M$ in the Grothendieck group $K(\mathcal{A})$ is denoted by $\widehat{M}$. Let $\mathcal{K}_{\geq 0}(\mathcal{A}) \subset K(\mathcal{A})$ be the subset of $K(\mathcal{A})$ consisting of the classes $\widehat{A} \in K(\mathcal{A})$ of $A \in \mathcal{A}$ (rather than the formal differences of them).

For an abelian category $\mathcal{A}$ which is essentially small, the set of its isomorphism classes is denoted by $\operatorname{Iso}(\mathcal{A})$.

For a complex $M_{\bullet}=\left(\cdots \rightarrow M_{i} \stackrel{d_{i}}{\longrightarrow} M_{i+1} \rightarrow \cdots\right)$ in an abelian category $\mathcal{A}$, its homology is denoted by $H_{*}\left(M_{\bullet}\right)$.

For a set $S$, we denote by $|S|$ its cardinality.

Date: July 4, 2012.

The author is supported by JSPS Fellowships for Young Scientists (No. 21-2241, 24-4759). 


\section{Hall algebras of COMplexes}

1.1. Hall algebra. This subsection gives basic definitions and properties of Hall algebra, following [1, $\S \S 2.3-$ $2.5]$ and [11, §1]. Let $\mathcal{A}$ be an abelian category satisfying the assumptions

(a) essentially small with finite morphism spaces,

(b) linear over $\mathfrak{k}$

(c') of finite global dimension.

Remark 1.1. We will introduce additional conditions (c), (d) and (e) in the following discussion. These symbols except (c') follow those in [1].

1.1.1. Definitions. Consider a vector space

$$
\mathcal{H}(\mathcal{A}):=\bigoplus_{A \in \operatorname{Iso}(\mathcal{A})} \mathbb{C}[A]
$$

linearly spanned by symbols $[A]$ with $A$ running through the $\operatorname{set} \operatorname{Iso}(\mathcal{A})$ of isomorphism classes of objects in $\mathcal{A}$.

Definition/Fact 1.2 (Ringel [10]). The following operation defines on $\mathcal{H}(\mathcal{A})$ the structure of a unital associative algebra over $\mathbb{C}$ :

$$
[A] \diamond[B]:=\sum_{C \in \operatorname{Iso}(\mathcal{A})} \frac{\left|\operatorname{Ext}_{\mathcal{A}}^{1}(A, B)_{C}\right|}{\left|\operatorname{Hom}_{\mathcal{A}}(A, B)\right|} \cdot[C] .
$$

Here

$$
\operatorname{Ext}_{\mathcal{A}}^{1}(A, B)_{C} \subset \operatorname{Ext}_{\mathcal{A}}^{1}(A, B)
$$

is the set parametrizing extensions of $B$ by $A$ with the middle term isomorphic to $C$.

The unit is given by [0], where 0 is the zero object of $\mathcal{A}$.

This algebra $(\mathcal{H}(\mathcal{A}), \diamond,[0])$ is called the Hall algebra of $\mathcal{A}$. Below we will denote it by $\mathcal{H}(\mathcal{A})$ for simplicity.

Remark 1.3. We follow [1] to choose $\left|\operatorname{Ext}_{\mathcal{A}}^{1}(A, B)_{C}\right| /\left|\operatorname{Hom}_{\mathcal{A}}(A, B)\right|$ for the structure constant of the multiplication. It is proportional to the usual structure constant $\left|\left\{B^{\prime} \subset C \mid B^{\prime} \cong B, C / B^{\prime} \cong A\right\}\right|$ appearing in [10] and [11. See [1, §2.3] for the detail.

1.1.2. Euler form and extended Hall algebra. Let us recall the notations for Grothendieck group given in \$0.2. For objects $A, B \in \mathcal{A}$, the Euler form is defined by

$$
\langle A, B\rangle:=\sum_{i \in \mathbb{Z}}(-1)^{i} \operatorname{dim}_{\mathfrak{k}} \operatorname{Ext}_{\mathcal{A}}^{i}(A, B)
$$

where the sum is finite by our assumptions on $\mathcal{A}$. As is well known, this form descends to the one on the Grothendieck group $K(\mathcal{A})$ of $\mathcal{A}$, which is denoted by the same symbol as (1.2):

$$
\langle\cdot, \cdot\rangle: K(\mathcal{A}) \times K(\mathcal{A}) \longrightarrow \mathbb{Z}
$$

We will also use the symmetrized Euler form:

$$
(\cdot, \cdot): K(\mathcal{A}) \times K(\mathcal{A}) \longrightarrow \mathbb{Z}, \quad(\alpha, \beta):=\langle\alpha, \beta\rangle+\langle\beta, \alpha\rangle .
$$

Definition/Fact 1.4. (1) The twisted Hall algebra $\mathcal{H}_{\mathrm{tw}}(\mathcal{A})$ is the same vector space as $\mathcal{H}(\mathcal{A})$ with the twisted multiplication

$$
[A] *[B]:=t^{\langle\widehat{A}, \widehat{B}\rangle} \cdot[A] \diamond[B]
$$

for $A, B \in \operatorname{Iso}(\mathcal{A})$. Here $t:=\sqrt{q}$ is the fixed square root of $q$.

(2) The extended Hall algebra $\widetilde{\mathcal{H}}(\mathcal{A})$ is defined as an extension of $\mathcal{H}_{\mathrm{tw}}(\mathcal{A})$ by adjoining symbols $K_{\alpha}$ for classes $\alpha \in K(\mathcal{A})$, and imposing relations

$$
K_{\alpha} * K_{\beta}=K_{\alpha+\beta}, \quad K_{\alpha} *[B]=t^{(\alpha, \widehat{B})} \cdot[B] * K_{\alpha}
$$

for $\alpha, \beta \in K(\mathcal{A})$ and $B \in \operatorname{Iso}(\mathcal{A})$. Note that $\widetilde{\mathcal{H}}(\mathcal{A})$ has a vector space basis consisting of the elements $K_{\alpha} *[B]$ for $\alpha \in K(\mathcal{A})$ and $B \in \operatorname{Iso}(\mathcal{A})$.

Remark 1.5. In [1], the extended Hall algebra is denoted by $\mathcal{H}_{\mathrm{tw}}^{\mathrm{e}}(\mathcal{A})$. 
1.1.3. Green's coproduct. To introduce a coalgebra structure, one should consider a completion of the algebra. Assume that the abelian category $\mathcal{A}$ satisfies the conditions (a), (b), (c') and

(e) nonzero object defines nonzero class in the Grothendieck group.

Then the algebra $\mathcal{H}(\mathcal{A})$ is naturally graded by the Grothendieck group $K(\mathcal{A})$ of $\mathcal{A}$ :

$$
\mathcal{H}(\mathcal{A})=\bigoplus_{\alpha \in K(\mathcal{A})} \mathcal{H}(\mathcal{A})[\alpha], \quad \mathcal{H}(\mathcal{A})[\alpha]:=\bigoplus_{\widehat{A}=\alpha} \mathbb{C}[A] .
$$

For $\alpha, \beta \in K(\mathcal{A})$, set

$$
\begin{aligned}
& \mathcal{H}(\mathcal{A})[\alpha] \widehat{\otimes}_{\mathbb{C}} \mathcal{H}(\mathcal{A})[\beta]:=\prod_{\widehat{A}=\alpha, \widehat{B}=\beta} \mathbb{C}[A] \otimes_{\mathbb{C}} \mathbb{C}[B], \\
& \mathcal{H}(\mathcal{A}) \widehat{\otimes}_{\mathbb{C}} \mathcal{H}(\mathcal{A}):=\prod_{\alpha, \beta \in K(\mathcal{A})} \mathcal{H}(\mathcal{A})[\alpha] \widehat{\otimes}_{\mathbb{C}} \mathcal{H}(\mathcal{A})[\beta] .
\end{aligned}
$$

Thus $\mathcal{H}(\mathcal{A}) \widehat{\otimes} \mathcal{H}(\mathcal{A})$ is the space of all formal linear combinations

$$
\sum_{A, B} c_{A, B} \cdot[A] \otimes[B] .
$$

This tensor product $\widehat{\otimes}$ is called a completed tensor product.

Definition/Fact 1.6. (1) (Green [7]) The following maps

$$
\Delta: \mathcal{H}(\mathcal{A}) \longrightarrow \mathcal{H}(\mathcal{A}) \widehat{\otimes}_{\mathbb{C}} \mathcal{H}(\mathcal{A}), \quad \epsilon: \mathcal{H}(\mathcal{A}) \longrightarrow \mathbb{C}
$$

define a topological coassociative coalgebra structure on $\mathcal{H}(\mathcal{A})$ :

$$
\Delta([A]):=\sum_{B, C} t^{\langle B, C\rangle} \frac{\left|\operatorname{Ext}_{\mathcal{A}}(B, C)_{A}\right|}{\left|\operatorname{Aut}_{\mathcal{A}}(A)\right|} \cdot[B] \otimes[C], \quad \epsilon([A]):=\delta_{A, 0}
$$

(2) (Xiao [14]) On the extended algebra, defining the maps

$$
\Delta: \widetilde{\mathcal{H}}(\mathcal{A}) \longrightarrow \widetilde{\mathcal{H}}(\mathcal{A}) \widehat{\otimes}_{\mathbb{C}} \widetilde{\mathcal{H}}(\mathcal{A}), \quad \epsilon: \widetilde{\mathcal{H}}(\mathcal{A}) \longrightarrow \mathbb{C}
$$

by (1.3) and

$$
\Delta\left(K_{\alpha}\right):=K_{\alpha} \otimes K_{\alpha}, \quad \epsilon\left(K_{\alpha}\right):=1,
$$

one has a topological coassociative coalgebra structure on $\widetilde{\mathcal{H}}(\mathcal{A})$.

Here the word topological means that everything should be considered in the completed space. For example, the coassociativity in (1) means that the two maps $(\Delta \otimes 1) \otimes \Delta$ and $(1 \otimes \Delta) \otimes \Delta$ from $\mathcal{H}(\mathcal{A})$ to $\mathcal{H}(\mathcal{A}) \widehat{\otimes} \mathcal{H}(\mathcal{A}) \widehat{\otimes} \mathcal{H}(\mathcal{A})$ coincide.

1.1.4. Bialgebra structure and Hopf pairing. Now we have an algebra structure and a coalgebra structure on $\mathcal{H}(A)$ (and on $\widetilde{\mathcal{H}}(A)$ ). In order that these structures are compatible and give a bialgebra structure, we must impose one more condition on $\mathcal{A}$.

Fact 1.7 (Green [7, Xiao [14). Assume that the abelian category $\mathcal{A}$ satisfies the conditions (a), (b), (c'), (e) and

(d) hereditary, that is, of global dimension at most 1.

Then the tuples

$$
(\mathcal{H}(\mathcal{A}), \diamond,[0], \Delta, \epsilon), \quad(\widetilde{\mathcal{H}}(\mathcal{A}), *,[0], \Delta, \epsilon)
$$

are topological bialgebras defined over $\mathbb{C}$. That is, the map $\Delta: \mathcal{H}(\mathcal{A}) \rightarrow \mathcal{H}(\mathcal{A}) \widehat{\otimes} \mathcal{H}(\mathcal{A})$ and $\Delta: \widetilde{\mathcal{H}}(\mathcal{A}) \rightarrow$ $\widetilde{\mathcal{H}}(\mathcal{A}) \widehat{\otimes} \widetilde{\mathcal{H}}(\mathcal{A})$ are homomorphisms of $\mathbb{C}$-algebras.

Below, we simply denote by $\mathcal{H}(\mathcal{A})$ and $\widetilde{\mathcal{H}}(\mathcal{A})$ the bialgebras $(\mathcal{H}(\mathcal{A}), \diamond,[0], \Delta, \epsilon)$ and $(\widetilde{\mathcal{H}}(\mathcal{A}), *,[0], \Delta, \epsilon)$ respectively.

This bialgebra structure on $\mathcal{H}(\mathcal{A})$ has an additional feature, that is, it is self-dual. The self-duality is stated in terms of a natural nondegenerate bilinear form, called Hopf pairing.

Definition/Fact 1.8 (Green, [7). Assume that the abelian cateogry $\mathcal{A}$ satisfies the conditions (a), (b), $\left(c^{\prime}\right),(d)$ and (e). 
(1) The non-degenerate bilinear form

$$
(\cdot, \cdot)_{H}: \mathcal{H}(\mathcal{A}) \otimes_{\mathbb{C}} \mathcal{H}(\mathcal{A}) \longrightarrow \mathbb{C}
$$

given by

$$
([A],[B])_{H}:=\frac{\delta_{A, B}}{\left|\operatorname{Aut}_{\mathcal{A}}(A)\right|}
$$

is a Hopf pairing on the bialgebra $\mathcal{H}(\mathcal{A})$, that is, for any $x, y, z \in \operatorname{Iso}(\mathcal{A})$, one has

$$
(x \diamond y, z)_{H}=(x \otimes y, z)_{H} .
$$

(2) The non-degenerate bilinear form

$$
(\cdot, \cdot)_{H}: \widetilde{\mathcal{H}}(\mathcal{A}) \otimes_{\mathbb{C}} \widetilde{\mathcal{H}}(\mathcal{A}) \longrightarrow \mathbb{C}
$$

given by

$$
\left([A] K_{\alpha},[B] K_{\beta}\right)_{H}:=\frac{\delta_{A, B}}{\left|\operatorname{Aut}_{\mathcal{A}}(A)\right|} t^{(\alpha, \beta)}
$$

is a Hopf pairing on the bialgebra $\widetilde{\mathcal{H}}(\mathcal{A})$, that is, for any $x, y, z \in \operatorname{Iso}(\mathcal{A})$, one has

$$
(x * y, z)_{H}=(x \otimes y, z)_{H} .
$$

Remark 1.9. In the right hand sides of (1.4) and (1.5), we used the usual pairing on the product space:

$$
(x \otimes y, z \otimes w)_{H}:=(x, z)_{H} \cdot(y, w)_{H}
$$

1.1.5. Drinfeld double. Here we recall the Drinfeld double of the self-dual bialgebra. For the complete treatment of Drinfeld double construction, we refer [9, §3.2] and [11, §5.2].

Fact 1.10 (Drinfeld). Let $\mathcal{H}$ be a $\mathbb{C}$-bialgebra with a Hopf pairing $(\cdot, \cdot)_{H}: \mathcal{H} \otimes_{\mathbb{C}} \mathcal{H} \rightarrow \mathbb{C}$. Then there is a unique algebra structure $\circ$ on $\mathcal{H} \otimes \mathcal{H}$ satisfying the following conditions

(1) The maps

$$
\mathcal{H} \longrightarrow \mathcal{H} \otimes_{\mathbb{C}} \mathcal{H}, \quad a \longmapsto a \otimes 1
$$

and

$$
\mathcal{H} \longrightarrow \mathcal{H} \otimes_{\mathbb{C}} \mathcal{H}, \quad a \longmapsto 1 \otimes a
$$

are injective homomorphisms of $\mathbb{C}$-algebras.

(2) For all elements $a, b \in \mathcal{H}$, one has

$$
(a \otimes 1) \circ(1 \otimes b)=a \otimes b .
$$

(3) For all elements $a, b \in \mathcal{H}$, one has

$$
\sum\left(a_{(2)}, b_{(2)}\right)_{H} \cdot a_{(1)} \otimes b_{(1)}=\sum\left(a_{(1)}, b_{(1)}\right)_{H} \cdot\left(1 \otimes b_{(2)}\right) \circ\left(a_{(2)} \otimes 1\right) .
$$

Here we used Sweedler's notation: $\Delta(a)=\sum a_{(1)} \otimes a_{(2)}$.

Remark 1.11. If $\mathcal{H}$ is a topological bialgebra, then one should replace the tensor product $\otimes$ in the statement by the completed one $\widehat{\otimes}$.

1.2. Hall algebras of complexes. We summarize necessary definitions and properties of Hall algebras of $\mathbb{Z}_{2}$-graded complexes. Most of the materials were introduced or shown in [1].

In this subsection $\$ 1.2, \mathcal{A}$ denotes an abelian category satisfying the following three conditions.

(a) essentially small with finite morphism spaces,

(b) linear over $\mathfrak{k}$,

(c) of finite global dimension and having enough projectives.

1.2.1. Categories of two-periodic complexes. We shall recall the basic definitions in [1, §3.1]. Let $\mathcal{C}_{\mathbb{Z}_{2}}(\mathcal{A})$ be the abelian category of $\mathbb{Z}_{2}$-graded complexes in $\mathcal{A}$. An object $M_{\bullet}$ of this category consists of the following diagram in $\mathcal{A}$ :

$$
M_{1} \underset{d_{0}}{\stackrel{d_{1}}{\rightleftarrows}} M_{0}, \quad d_{i+1} \circ d_{i}=0
$$

Hereafter indices in the diagram of an object in $\mathcal{C}_{\mathbb{Z}_{2}}(\mathcal{A})$ are understood by modulo 2 . A morphism $s_{\bullet}: M_{\bullet} \rightarrow$ $N$. consists of a diagram

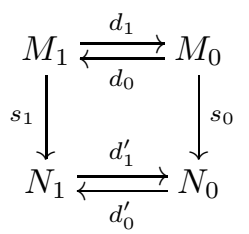


with $s_{i+1} \circ d_{i}=d_{i}^{\prime} \circ s_{i}$. Two morphisms $s_{\bullet}, t_{\bullet}: M_{\bullet} \rightarrow N_{\bullet}$ are said to be homotopic if there are morphisms $h_{i}: M_{i} \rightarrow N_{i+1}$ such that

$$
t_{i}-s_{i}=d_{i+1}^{\prime} \circ h_{i}+h_{i+1} \circ d_{i} .
$$

For an object $M_{\bullet} \in C_{\mathbb{Z}_{2}}(\mathcal{A})$, we define its class in the $K$-group by

$$
\widehat{M}_{\bullet}:=\widehat{M}_{0}-\widehat{M}_{1} \in K(\mathcal{A}) \text {. }
$$

Denote by $\operatorname{Ho}_{\mathbb{Z}_{2}}(\mathcal{A})$ the category obtained from $\mathcal{C}_{\mathbb{Z}_{2}}(\mathcal{A})$ by identifying homotopic morphisms. Let us also denote by

$$
\mathcal{C}_{\mathbb{Z}_{2}}(\mathcal{P}) \subset \mathcal{C}_{\mathbb{Z}_{2}}(\mathcal{A})
$$

the full subcategories whose objects are complexes of projectives in $\mathcal{A}$. Hereafter we drop the subscript $\mathbb{Z}_{2}$ and just write

$$
\mathcal{C}(\mathcal{A}):=\mathcal{C}_{\mathbb{Z}_{2}}(\mathcal{A}), \quad \mathcal{C}(\mathcal{P}):=\mathcal{C}_{\mathbb{Z}_{2}}(\mathcal{P}), \quad \operatorname{Ho}(\mathcal{A}):=\operatorname{Ho}_{\mathbb{Z}_{2}}(\mathcal{A})
$$

The shift functor [1] of complexes induces an involution

$$
\mathcal{C}(\mathcal{A}) \stackrel{*}{\longleftrightarrow} \mathcal{C}(\mathcal{A})
$$

This involution shifts the grading and changes the sign of the differential as follows:

$$
M_{\bullet}=\left(M_{1} \underset{d_{0}}{\stackrel{d_{1}}{\rightleftarrows}} M_{0}\right) \stackrel{*}{\longleftrightarrow} M_{\bullet}^{*}=\left(M_{0} \underset{-d_{1}}{\stackrel{-d_{0}}{\rightleftarrows}} M_{1}\right)
$$

Now let us recall

Fact $1.12\left(\right.$ [1, Lemma 3.3]). For $M_{\bullet}, N_{\bullet} \in \mathcal{C}(\mathcal{P})$ we have

$$
\operatorname{Ext}_{\mathcal{C}(\mathcal{A})}^{1}\left(N_{\bullet}, M_{\bullet}\right) \cong \operatorname{Hom}_{\mathrm{Ho}(\mathcal{A})}\left(N_{\bullet}, M_{\bullet}^{*}\right) .
$$

A complex $M_{\bullet} \in \mathcal{C}(\mathcal{A})$ is called acyclic if $H_{*}\left(M_{\bullet}\right)=0$. To each object $P \in \mathcal{P}$, we can attach acyclic complexes

$$
K_{P \bullet}=(P \underset{0}{\stackrel{\mathrm{Id}}{\rightleftarrows}} P), \quad K_{P}^{*}=(P \underset{-\mathrm{Id}}{\stackrel{0}{\rightleftarrows}} P)
$$

Remark 1.13. The complexes $K_{P}, K_{P}^{*}$ are denoted by $K_{P}, K_{P}^{*}$ in [1].

Let us recall the following fact shown in [1].

Fact 1.14 ([1, Lemma 3.2]). For each acyclic complex of projectives $M_{\bullet} \in \mathcal{C}(\mathcal{P})$, there are objects $P, Q \in \mathcal{P}$, unique up to isomorphism, such that $M_{\bullet} \cong K_{P} \oplus K_{Q}$ *.

1.2.2. Definition of Hall algebras of complexes. Let $\mathcal{H}(\mathcal{C}(\mathcal{A}))$ be the Hall algebra of the abelian category $\mathcal{C}(\mathcal{A})$ defined in $§ 1.1$. As noted in [1, §3.5], this definition makes sense since the spaces $\operatorname{Ext}_{\mathcal{C}(\mathcal{A})}^{1}\left(N_{\bullet}, M_{\bullet}\right)$ are all finite-dimensional by Fact 1.12, Let

$$
\mathcal{H}(\mathcal{C}(\mathcal{P})) \subset \mathcal{H}(\mathcal{C}(\mathcal{A}))
$$

be the subspace spanned by complexes of projective objects.

Define $\mathcal{H}_{\text {tw }}(\mathcal{C}(\mathcal{P}))$ to be the same vector space as $\mathcal{H}(\mathcal{C}(\mathcal{P})$ ) with the twisted multiplication

$$
\left[M_{\bullet}\right] *\left[N_{\bullet}\right]:=t^{\left\langle M_{0}, N_{0}\right\rangle+\left\langle M_{1}, N_{1}\right\rangle} \cdot\left[M_{\bullet}\right] \diamond\left[N_{\bullet}\right] .
$$

Now let us recall the simple relations satisfied by the acyclic complexes $K_{P}$ :

Fact 1.15 ([1, Lemmas 3.4,3.5]). For any object $P \in \mathcal{P}$ and any complex $M_{\bullet} \in \mathcal{C}(\mathcal{P})$, we have the following relations in $\mathcal{H}_{\mathrm{tw}}(\mathcal{C}(\mathcal{P}))$ :

$$
\begin{aligned}
& {\left[K_{P_{\bullet}}\right] *\left[M_{\bullet}\right]=t^{\left\langle\widehat{P}, \widehat{M}_{\bullet}\right\rangle} \cdot\left[K_{P} \oplus M_{\bullet}\right], \quad\left[M_{\bullet}\right] *\left[K_{P}\right]=t^{-\left\langle\widehat{M}_{\bullet}, \widehat{P}\right\rangle} \cdot\left[K_{P} \oplus M_{\bullet}\right],} \\
& {\left[K_{P_{\bullet}}\right] *\left[M_{\bullet}\right]=t^{\left(\widehat{P}, \widehat{M}_{\bullet}\right)} \cdot\left[M_{\bullet}\right] *\left[K_{P_{\bullet}}\right], \quad\left[K_{P_{\bullet}}^{*}\right] *\left[M_{\bullet}\right]=t^{-\left(\widehat{P}, \widehat{M}_{\bullet}\right)} \cdot\left[M_{\bullet}\right] *\left[K_{P}{ }^{*}\right]}
\end{aligned}
$$

In particular, for $P, Q \in \mathcal{P}$ we have

$$
\begin{aligned}
& {\left[K_{P_{\mathbf{\bullet}}}\right] *\left[K_{Q_{\mathbf{\bullet}}}\right]=\left[K_{P_{\bullet}} \oplus K_{Q_{\mathbf{.}}}\right], \quad\left[K_{P_{\bullet}}\right] *\left[K_{Q_{\bullet}^{*}}^{*}\right]=\left[K_{P} \oplus K_{Q_{\bullet}}^{*}\right],} \\
& {\left[\left[K_{P_{\mathbf{\bullet}}}\right],\left[K_{Q_{\bullet}}\right]\right]=\left[\left[K_{P_{\bullet}}\right],\left[K_{Q_{\bullet}}^{*}\right]\right]=\left[\left[K_{P_{\bullet}^{*}}^{*}\right],\left[K_{Q_{\bullet}^{*}}^{*}\right]\right]=0 .}
\end{aligned}
$$

At the last line we used the commutator $[x, y]:=x * y-y * x$. 
1.2.3. Bridgeland's Hall algebra. Now we can introduce the main object: Bridgeland's Hall algebra. We define the localized Hall algebra $\mathcal{D H}(\mathcal{A})$ to be the localization of $\mathcal{H}_{\mathrm{tw}}(\mathcal{C}(\mathcal{P})$ ) with respect to the elements $\left[M_{\bullet}\right]$ corresponding to acyclic complexes $M_{\bullet}$ :

$$
\mathcal{D H}(\mathcal{A}):=\mathcal{H}_{\text {tw }}(\mathcal{C}(\mathcal{P}))\left[\left[M_{\bullet}\right]^{-1} \mid H_{*}\left(M_{\bullet}\right)=0\right]
$$

As explained in [1, $\S 3.6]$, this is the same as localizing by the elements $\left[K_{P}\right]$ and $\left[K_{P}^{*}\right]$ for all objects $P \in \mathcal{P}$. For an element $\alpha \in K(\mathcal{A})$, we define

$$
K_{\alpha}:=\left[K_{P}\right] *\left[K_{Q}\right]^{-1}, \quad K_{\alpha}^{*}:=\left[K_{P}^{*}\right] *\left[K_{Q}^{*}\right]^{-1},
$$

where we expressed $\alpha=\widehat{P}-\widehat{Q}$ using the classes of some projectives $P, Q \in \mathcal{P}$. This is well defined by Fact 1.15

Remark 1.16. We will denote two different elements $K_{\alpha} \in \widetilde{\mathcal{H}}(\mathcal{A})$ and $K_{\alpha} \in \mathcal{D} \mathcal{H}(\mathcal{A})$ by the same symbol, following [1].

By Fact 1.15, we immediately have

Corollary 1.17. In the algebra $\mathcal{D H}(\mathcal{A})$, we have

$$
K_{\alpha} * M_{\bullet}=t^{\left(\alpha, \widehat{M}_{\bullet}\right)} \cdot M_{\bullet} * K_{\alpha}, \quad K_{\alpha}^{*} * M_{\bullet}=t^{-\left(\alpha, \widehat{M}_{\bullet}\right)} \cdot M_{\bullet} * K_{\alpha},
$$
for arbitrary $\alpha \in K(\mathcal{A})$ and $M_{\bullet} \in \mathcal{C}(\mathcal{P})$.

for arbitrary $\alpha, \beta \in K(\mathcal{A})$.

$$
\left[K_{\alpha}, K_{\beta}\right]=\left[K_{\alpha}, K_{\beta}^{*}\right]=\left[K_{\alpha}^{*}, K_{\beta}^{*}\right]=0
$$

1.3. Hereditary case. In this subsection $₫ 1.3$, we assume that $\mathcal{A}$ satisfies the conditions (a), (b), (c) and the following additional ones:

(d) $\mathcal{A}$ is hereditary, that is of global dimension at most 1 ,

(e) nonzero objects in $\mathcal{A}$ define nonzero classes in $K(\mathcal{A})$.

Then by [1, §4] we have a nice basis for $\mathcal{D H}(\mathcal{A})$. To explain that, let us recall the minimal resolution of objects of $\mathcal{A}$.

1.3.1. Minimal resolution and the complex $C_{A}$.

Definition $1.18([1, \S 4.1])$. Assume the conditions (a),(c),(d) on $\mathcal{A}$. Then every object $A \in \mathcal{A}$ has a projective resolution

$$
0 \rightarrow P \stackrel{f}{\rightarrow} Q \stackrel{g}{\rightarrow} A \rightarrow 0,
$$

and decomposing $P$ and $Q$ into finite direct sums $P=\oplus_{i} P_{i}, Q=\oplus_{j} Q_{j}$, one may write $f=\left(f_{i j}\right)$ in matrix form with $f_{i j}: P_{i} \rightarrow Q_{j}$. The resolution (1.9) is said to be minimal if none of the morphisms $f_{i j}$ is an isomorphism.

Fact 1.19 (1, Lemma 4.1]). Any resolution (1.9) is isomorphic to a resolution of the form

$$
0 \rightarrow R \oplus P^{\prime} \stackrel{1 \oplus f^{\prime}}{\longrightarrow} R \oplus Q^{\prime} \stackrel{\left(0, g^{\prime}\right)}{\longrightarrow} A \rightarrow 0
$$

with some object $R \in \mathcal{P}$ and some minimal projective resolution

$$
0 \rightarrow P^{\prime} \stackrel{f^{\prime}}{\rightarrow} Q^{\prime} \stackrel{g^{\prime}}{\rightarrow} A \rightarrow 0 .
$$

Definition $1.20([1, \S 4.2])$. Given an object $A \in \mathcal{A}$, take a minimal projective resolution

$$
0 \rightarrow P_{A} \stackrel{f_{A}}{\longrightarrow} Q_{A} \stackrel{g}{\rightarrow} A \rightarrow 0,
$$

We define a $\mathbb{Z}_{2}$-graded complex

$$
C_{A_{\bullet}}:=\left(P_{A} \stackrel{f_{A}}{\rightleftarrows} Q_{A}\right) \in \mathcal{C}(\mathcal{P})
$$

Remark 1.21. The complex $C_{A}$. is denoted as $C_{A}$ in [1].

By Fact 1.19, arbitrary two minimal projective resolutions of $A$ are isomorphic, so the complex $C_{A}$. is well-defined up to isomorphism.

Fact 1.22 ([1, Lemma 4.2]). Every object $M_{\bullet} \in C(\mathcal{P})$ has a direct sum decomposition

$$
M_{\bullet}=C_{A} \oplus C_{B}^{*} \oplus K_{P \bullet} \oplus K_{Q_{\bullet}^{*}}^{*}
$$

Moreover, the objects $A, B \in \mathcal{A}$ and $P, Q \in \mathcal{P}$ are unique up to isomorphism. 
1.3.2. Triangular decomposition.

Definition $1.23([1, \S \S 4.3-4.4])$. Given an object $A \in \mathcal{A}$, we define elements $E_{A}, F_{A} \in \mathcal{D} \mathcal{H}(\mathcal{A})$ by

$$
E_{A}:=t^{\langle\widehat{P}, \widehat{A}\rangle} \cdot K_{-\widehat{P}} *\left[C_{A}\right], \quad F_{A}:=E_{A}^{*} .
$$

Here we used a projective decomposition (1.10) of $A$ and the associated complex $C_{A}$. in Definition 1.20 .

Fact 1.24 ([1, Lemmas 4.6, 4.7]). (1) There is an embedding of algebras

$$
I_{+}^{\mathrm{e}}: \tilde{\mathcal{H}}(\mathcal{A}) \longleftrightarrow \mathcal{D} \mathcal{H}(\mathcal{A})
$$

defined by

$$
[A] \longmapsto E_{A}(A \in \operatorname{Iso}(\mathcal{A})), \quad K_{\alpha} \longmapsto K_{\alpha}(\alpha \in K(\mathcal{A})) \text {. }
$$

By composing $I_{+}^{\mathrm{e}}$ and the involution $*$, we also have an embedding

$$
I_{-}^{\mathrm{e}}: \widetilde{\mathcal{H}}(\mathcal{A}) \longleftrightarrow \mathcal{D} \mathcal{H}(\mathcal{A})
$$

defined by

$$
[A] \longmapsto F_{A}(A \in \operatorname{Iso}(\mathcal{A})), \quad K_{\alpha} \longmapsto K_{\alpha}^{*}(\alpha \in K(\mathcal{A})) .
$$

(2) The multiplication map

$$
m: a \otimes b \longmapsto I_{+}^{\mathrm{e}}(a) * I_{-}^{\mathrm{e}}(b)
$$

defines an isomorphism of vector spaces

$$
m: \widetilde{\mathcal{H}}(\mathcal{A}) \otimes_{\mathbb{C}} \tilde{\mathcal{H}}(\mathcal{A}) \stackrel{\sim}{\longrightarrow} \mathcal{D H}(\mathcal{A}) .
$$

As a corollary, we have

Corollary 1.25. $\mathcal{D H}(\mathcal{A})$ has a basis consisting of elements

$$
E_{A} * K_{\alpha} * K_{\beta}^{*} * F_{B}, \quad A, B \in \operatorname{Iso}(\mathcal{A}), \alpha, \beta \in K(\mathcal{A}) .
$$

1.4. Main theorem. Now we can state our main theorem.

Theorem 1.26. Assume that the abelian category $\mathcal{A}$ satisfies the conditions (a)-(e). Then the algebra $\mathcal{D H}(\mathcal{A})$ is isomorphic to the Drinfeld double of the bialgebra $\widetilde{\mathcal{H}}(\mathcal{A})$.

\section{Proof OF THE MAIN THEOREM}

Because of the description of the basis of $\mathcal{D H}(\mathcal{A})$ (Corollary 1.25) and the definition of Drinfeld double (Fact 1.10), the proof of Theorem 1.26 is reduced to check the equation (1.6) for the elements consisting of the basis of $\widetilde{\mathcal{H}}(\mathcal{A})$.

Let us write the equation (1.6) in the present situation:

$$
\sum\left(a_{(2)}, b_{(2)}\right)_{H} \cdot I_{+}^{\mathrm{e}}\left(a_{(1)}\right) * I_{-}^{\mathrm{e}}\left(b_{(1)}\right) \stackrel{?}{=} \sum\left(a_{(1)}, b_{(1)}\right)_{H} \cdot I_{-}^{\mathrm{e}}\left(b_{(2)}\right) * I_{+}^{\mathrm{e}}\left(a_{(2)}\right) .
$$

What we must do is to check the equation for the cases

$$
\text { (1) }(a, b)=\left(K_{\alpha}, K_{\beta}\right) \text {, }
$$

(2) $(a, b)=\left([A], K_{\beta}\right)$,

$\left(2^{\prime}\right)(a, b)=\left(K_{\alpha},[B]\right)$

(3) $(a, b)=([A],[B])$

with arbitrary $\alpha, \beta \in K(\mathcal{A})$ and $A, B \in \operatorname{Iso}(\mathcal{A})$.

2.1. Case (1). It is easy to check the equation (2.1) for the case (1) in (2.2). Since $\Delta\left(K_{\alpha}\right)=K_{\alpha} \otimes K_{\alpha}$, the equation in this case becomes

$$
\left(K_{\alpha}, K_{\beta}\right)_{H} \cdot K_{\alpha} * K_{\beta}^{*} \stackrel{?}{=}\left(K_{\alpha}, K_{\beta}\right)_{H} \cdot K_{\beta}^{*} * K_{\alpha},
$$

which is valid by Corollary 1.17 (2). 
2.2. Cases (2) and (2'). The cases (2) and (2') in (2.2) are trivial. In fact, for the case (2), we may write

$$
\Delta([A])=\sum_{A_{1}, A_{2}} g_{A_{1}, A_{2}}^{A} \cdot\left[A_{1}\right] \otimes\left[A_{2}\right]
$$

with some $g_{A_{1}, A_{2}}^{A} \in \mathbb{C}$ and $\Delta\left(K_{\beta}\right)=K_{\beta} \otimes K_{\beta}$. Then (2.1) reads

$$
\sum_{A_{1}, A_{2}}\left(\left[A_{2}\right], K_{\beta}\right)_{H} g_{A_{1}, A_{2}}^{A} \cdot E_{A_{1}} * K_{\beta} \stackrel{?}{=} \sum_{A_{1}, A_{2}}\left(\left[A_{1}\right], K_{\beta}\right)_{H} g_{A_{1}, A_{2}}^{A} \cdot K_{\beta} * E_{A_{2}} .
$$

But recalling the Hopf pairing

$$
\left([A] K_{\alpha},[B] K_{\beta}\right)_{H}=\frac{\delta_{A, B}}{\left|\operatorname{Aut}_{\mathcal{A}}(A)\right|} t^{(\alpha, \beta)},
$$

in Definition/Fact 1.8, the equation reads

$$
g_{A, 0}^{A} \cdot E_{A} * K_{0} \stackrel{?}{=} g_{0, A}^{A} \cdot K_{0} * E_{A} .
$$

this equation trivially holds by Corollary $1.17(1)$ and $g_{A, 0}^{A}=g_{0, A}^{A}$. The case (2') is similar.

2.3. Case (3). The case (3) is non-trivial. Let us write

$$
\begin{aligned}
& \Delta([A])=\sum_{A_{1}, A_{2}} t^{\left\langle A_{1}, A_{2}\right\rangle} \frac{\left|\operatorname{Ext}_{\mathcal{A}}\left(A_{1}, A_{2}\right)_{A}\right|}{\left|\operatorname{Aut}_{\mathcal{A}}(A)\right|} \cdot\left[A_{1}\right] \otimes\left[A_{2}\right], \\
& \Delta([B])=\sum_{B_{1}, B_{2}} t^{\left\langle B_{1}, B_{2}\right\rangle} \frac{\left|\operatorname{Ext}_{\mathcal{A}}\left(B_{1}, B_{2}\right)_{B}\right|}{\left|\operatorname{Aut}_{\mathcal{A}}(B)\right|} \cdot\left[B_{1}\right] \otimes\left[B_{2}\right] .
\end{aligned}
$$

Then the equation (2.1) reads

$$
\begin{aligned}
& \sum_{A_{1}, A_{2}, B_{1}, B_{2}} t^{\left\langle A_{1}, A_{2}\right\rangle} \frac{\left|\operatorname{Ext}_{\mathcal{A}}\left(A_{1}, A_{2}\right)_{A}\right|}{\left|\operatorname{Aut}_{\mathcal{A}}(A)\right|} t^{\left\langle B_{1}, B_{2}\right\rangle} \frac{\left|\operatorname{Ext}_{\mathcal{A}}\left(B_{1}, B_{2}\right)_{B}\right|}{\left|\operatorname{Aut}_{\mathcal{A}}(B)\right|}\left(A_{2}, B_{2}\right)_{H} \cdot E_{A_{1}} * F_{B_{1}} \\
\stackrel{?}{=} & \sum_{A_{1}, A_{2}, B_{1}, B_{2}} t^{\left\langle A_{1}, A_{2}\right\rangle} \frac{\left|\operatorname{Ext}_{\mathcal{A}}\left(A_{1}, A_{2}\right)_{A}\right|}{\left|\operatorname{Aut}_{\mathcal{A}}(A)\right|} t^{\left\langle B_{1}, B_{2}\right\rangle} \frac{\left|\operatorname{Ext}_{\mathcal{A}}\left(B_{1}, B_{2}\right)_{B}\right|}{\left|\operatorname{Aut}_{\mathcal{A}}(B)\right|}\left(A_{1}, B_{1}\right)_{H} \cdot F_{B_{2}} * E_{A_{2}} .
\end{aligned}
$$

By the Hopf pairing (2.3), the left hand side of (2.4) becomes

$$
\begin{aligned}
\text { LHS of (2.4) } & =\sum_{A_{1}, A_{2}, B_{1}, B_{2}} t^{\left\langle A_{1}, A_{2}\right\rangle+\left\langle B_{1}, B_{2}\right\rangle} \frac{\left|\operatorname{Ext}_{\mathcal{A}}\left(A_{1}, A_{2}\right)_{A}\right|}{\left|\operatorname{Aut}_{\mathcal{A}}(A)\right|} \frac{\left|\operatorname{Ext}_{\mathcal{A}}\left(B_{1}, B_{2}\right)_{B}\right|}{\left|\operatorname{Aut}_{\mathcal{A}}(B)\right|} \frac{\delta_{A_{2}, B_{2}}}{\left|\operatorname{Aut}_{\mathcal{A}}\left(A_{2}\right)\right|} \cdot E_{A_{1}} * F_{B_{1}} \\
& =\sum_{A_{1}, A_{2}, B_{1}} t^{\left\langle A_{1}+B_{1}, A_{2}\right\rangle} \frac{\left|\operatorname{Ext}_{\mathcal{A}}\left(A_{1}, A_{2}\right)_{A}\right|}{\left|\operatorname{Aut}_{\mathcal{A}}(A)\right|} \frac{\left|\operatorname{Ext}_{\mathcal{A}}\left(B_{1}, A_{2}\right)_{B}\right|}{\left|\operatorname{Aut}_{\mathcal{A}}(B)\right|} \frac{1}{\left|\operatorname{Aut}_{\mathcal{A}}\left(A_{2}\right)\right|} \cdot E_{A_{1}} * F_{B_{1}} .
\end{aligned}
$$

By Definition 1.23, it becomes

$$
\begin{gathered}
=\sum_{A_{1}, A_{2}, B_{1}} t^{\left\langle A_{1}+B_{1}, A_{2}\right\rangle+\left\langle P_{A_{1}}, A_{1}\right\rangle+\left\langle P_{B_{1}}, B_{1}\right\rangle} \frac{\left|\operatorname{Ext}_{\mathcal{A}}\left(A_{1}, A_{2}\right)_{A}\right|}{\left|\operatorname{Aut}_{\mathcal{A}}(A)\right|} \frac{\left|\operatorname{Ext}_{\mathcal{A}}\left(B_{1}, A_{2}\right)_{B}\right|}{\left|\operatorname{Aut}_{\mathcal{A}}(B)\right|} \frac{1}{\left|\operatorname{Aut}_{\mathcal{A}}\left(A_{2}\right)\right|} \\
\cdot K_{-\widehat{P}_{A_{1}}} *\left[C_{A_{1}}\right] * K_{-\widehat{P}_{B_{1}}}^{*} *\left[C_{B_{1}}{ }^{*}\right] .
\end{gathered}
$$

By Corollary 1.17 (1), it becomes

$$
\begin{gathered}
=\sum_{A_{1}, A_{2}, B_{1}} t^{\left\langle A_{1}+B_{1}, A_{2}\right\rangle+\left\langle P_{A_{1}}, A_{1}\right\rangle+\left\langle P_{B_{1}}, B_{1}\right\rangle-\left(P_{B_{1}}, \widehat{C}_{A_{1}} \bullet\right.} \frac{\left|\operatorname{Ext}_{\mathcal{A}}\left(A_{1}, A_{2}\right)_{A}\right| \cdot\left|\operatorname{Ext}_{\mathcal{A}}\left(B_{1}, A_{2}\right)_{B}\right|}{\left|\operatorname{Aut}_{\mathcal{A}}(A)\right| \cdot\left|\operatorname{Aut}_{\mathcal{A}}(B)\right| \cdot\left|\operatorname{Aut}_{\mathcal{A}}\left(A_{2}\right)\right|} \\
\cdot K_{-\widehat{P}_{A_{1}}} * K_{-\widehat{P}_{B_{1}}}^{*} *\left[C_{A_{1}}\right] *\left[C_{B_{1}}{ }^{*}\right] .
\end{gathered}
$$

Since $\widehat{C}_{A_{1} \bullet}=\widehat{P}_{A_{1}}-\widehat{Q}_{A_{1}}=\widehat{A}$, it becomes

$$
\begin{gathered}
=\sum_{A_{1}, A_{2}, B_{1}} t^{\left\langle A_{1}+B_{1}, A_{2}\right\rangle+\left\langle P_{A_{1}}, A_{1}\right\rangle+\left\langle P_{B_{1}}, B_{1}\right\rangle-\left(P_{B_{1}}, A\right)} \frac{\left|\operatorname{Ext}_{\mathcal{A}}\left(A_{1}, A_{2}\right)_{A}\right| \cdot\left|\operatorname{Ext}_{\mathcal{A}}\left(B_{1}, A_{2}\right)_{B}\right|}{\left|\operatorname{Aut}_{\mathcal{A}}(A)\right| \cdot\left|\operatorname{Aut}_{\mathcal{A}}(B)\right| \cdot\left|\operatorname{Aut}_{\mathcal{A}}\left(A_{2}\right)\right|} \\
\cdot K_{-\widehat{P}_{A_{1}}} * K_{-\widehat{P}_{B_{1}}}^{*} *\left[C_{A_{1}}\right] *\left[C_{B_{1}}{ }^{*}\right] .
\end{gathered}
$$

Now by the definition of multiplication (1.1) and (1.7) in $\mathcal{D H}(\mathcal{A})$, one may write

$$
\left[C_{A_{1}}\right] *\left[C_{B_{1}}{ }^{*}\right]=\sum_{M_{\bullet} \in \operatorname{Iso}(\mathcal{C}(\mathcal{P}))} t^{\left\langle P_{A_{1}}, Q_{B_{1}}\right\rangle+\left\langle Q_{A_{1}}, P_{B_{1}}\right\rangle} \frac{\left|\operatorname{Ext}_{\mathcal{C}(\mathcal{A})}\left(C_{A_{1} \bullet}, C_{B_{1}}{ }^{*}\right)_{M_{\bullet}}\right|}{\left|\operatorname{Hom}_{\mathcal{C}(\mathcal{A})}\left(C_{A_{1} \bullet}, C_{B_{1}}{ }_{\bullet}\right)\right|} \cdot\left[M_{\bullet}\right] .
$$


Here the complex $M_{\bullet}$ sits in the commutative diagram

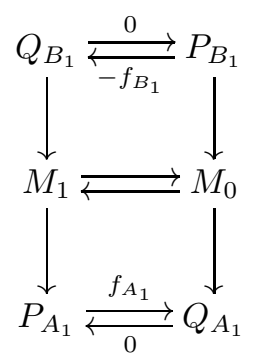

where both columns give short exact sequences in $\mathcal{A}$. Then, since $P_{A_{1}}$ and $Q_{A_{1}}$ are projective, we have $M_{1} \cong Q_{B_{1}} \oplus P_{A_{1}}$ and $M_{0} \cong P_{B_{1}} \oplus Q_{A_{1}}$.

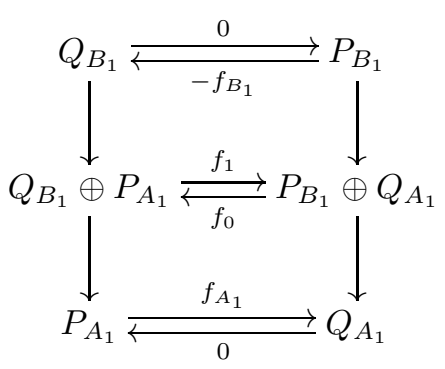

Now the commutativity of the diagram restricts the morphisms $M_{1} \rightarrow M_{0}$ and $M_{0} \rightarrow M_{1}$ of the following types:

$$
f_{1}=\left(\begin{array}{cc}
0 & s_{1} \\
0 & f_{A_{1}}
\end{array}\right), \quad f_{0}=\left(\begin{array}{cc}
-f_{B_{1}} & s_{0} \\
0 & 0
\end{array}\right)
$$

with $s_{1}: P_{A_{1}} \rightarrow P_{B_{1}}$ and $s_{0}: Q_{A_{1}} \rightarrow Q_{B_{1}}$ (see also the argument in the proof of [1, Lemma 3.3]).

Note also that

$$
\operatorname{Hom}_{\mathcal{C}(\mathcal{A})}\left(C_{A_{1} \bullet}, C_{B_{1} \bullet}^{*}\right) \cong \operatorname{Hom}_{\mathcal{A}}\left(P_{A_{1}}, Q_{B_{1}}\right)
$$

which can be easily check. Then the term $\left|\operatorname{Hom}_{\mathcal{C}(\mathcal{A})}\left(C_{A_{1} \bullet}, C_{B_{1}}{ }_{\bullet}^{*}\right)\right|$ at the denominator of $(2.6)$ is equal to

$$
\left|\operatorname{Hom}_{\mathcal{C}(\mathcal{A})}\left(C_{A_{1} \bullet}, C_{B_{1}}^{*} \bullet\right)\right|=\left|\operatorname{Hom}_{\mathcal{A}}\left(P_{A_{1}}, Q_{B_{1}}\right)\right|=t^{2\left\langle P_{A_{1}}, Q_{B_{1}}\right\rangle}
$$

At the last equation, we used the hereditary property of $\mathcal{A}$ and the projective property of $\mathcal{P}_{A_{1}}$ and $\mathcal{Q}_{B_{1}}$.

Combining (2.5), (2.6) and (2.8), the light hand side of (2.4) becomes

$$
\begin{gathered}
\text { LHS of (2.4) }=\sum_{A_{1}, A_{2}, B_{1}, M_{\bullet}} t^{\left\langle A_{1}+B_{1}, A_{2}\right\rangle+\left\langle P_{A_{1}}, A_{1}\right\rangle+\left\langle P_{B_{1}}, B_{1}\right\rangle-\left(P_{B_{1}}, P_{A_{1}}\right)+\left(P_{B_{1}}, Q_{A_{1}}\right)-\left\langle P_{A_{1}}, Q_{B_{1}}\right\rangle+\left\langle Q_{A_{1}}, P_{B_{1}}\right\rangle} \\
\cdot \frac{\left|\operatorname{Ext}_{\mathcal{A}}\left(A_{1}, A_{2}\right)_{A}\right| \cdot\left|\operatorname{Ext}_{\mathcal{A}}\left(B_{1}, A_{2}\right)_{B}\right| \cdot\left|\operatorname{Ext}_{\mathcal{C}(\mathcal{A})}\left(C_{A_{1}}, C_{B_{1}}{ }^{*}\right)_{M_{\bullet}}\right|}{\left|\operatorname{Aut}_{\mathcal{A}}(A)\right| \cdot\left|\operatorname{Aut}_{\mathcal{A}}(B)\right| \cdot\left|\operatorname{Aut}_{\mathcal{A}}\left(A_{2}\right)\right|} \\
\cdot K_{-\widehat{P}_{A_{1}} * K_{-\widehat{P}_{B_{1}}}^{*} *\left[M_{\bullet}\right]}
\end{gathered}
$$

A similar argument yields

$$
\begin{aligned}
& \text { RHS of }(\underline{2.4})=\sum_{\widetilde{A}_{1}, \widetilde{A}_{2}, \widetilde{B}_{2}, N_{\bullet}} t^{\left\langle\widetilde{A}_{1}, \widetilde{A}_{2}+\widetilde{B}_{2}\right\rangle+\left\langle P_{\widetilde{B}_{2}}, \widetilde{B}_{2}\right\rangle+\left\langle P_{\widetilde{A}_{2}}, \widetilde{A}_{2}\right\rangle-\left(P_{\widetilde{A}_{2}}, \widetilde{B}_{2}\right)+\left\langle Q_{\widetilde{B}_{2}}, P_{\widetilde{A}_{2}}\right\rangle-\left\langle P_{\widetilde{B}_{2}}, Q_{\widetilde{A}_{2}}\right\rangle} \\
& \frac{\left|\operatorname{Ext}_{\mathcal{A}}\left(\widetilde{A}_{1}, \widetilde{A}_{2}\right)_{A}\right| \cdot\left|\operatorname{Ext}_{\mathcal{A}}\left(\widetilde{A}_{1}, \widetilde{B}_{2}\right)_{B}\right| \cdot\left|\operatorname{Ext}_{\mathcal{C}(\mathcal{A})}\left(C_{\widetilde{B}_{2}}{ }^{*}, C_{\widetilde{A}_{2}}\right)_{N_{\bullet}}\right|}{\left|\operatorname{Aut}_{\mathcal{A}}(A)\right| \cdot\left|\operatorname{Aut}_{\mathcal{A}}(B)\right| \cdot\left|\operatorname{Aut}_{\mathcal{A}}\left(A_{1}\right)\right|} \\
& \cdot K_{-\widehat{P}_{\widetilde{A}_{2}}} * K_{-\widehat{P}_{\widetilde{B}_{2}}}^{*} *\left[N_{\bullet}\right]
\end{aligned}
$$


Here the complex $N_{\bullet}$ is of the next form:

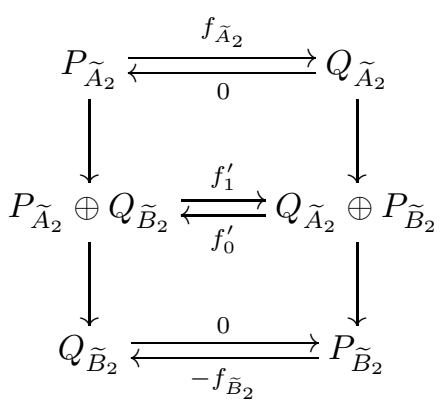

where

$$
f_{1}^{\prime}=\left(\begin{array}{cc}
f_{\widetilde{A}_{2}} & s_{1}^{\prime} \\
0 & 0
\end{array}\right), \quad f_{0}^{\prime}=\left(\begin{array}{cc}
0 & s_{0}^{\prime} \\
0 & -f_{\widetilde{B}_{2}}
\end{array}\right)
$$

with $s_{1}^{\prime}: Q_{\widetilde{B}_{2}} \rightarrow Q_{\widetilde{A}_{2}}$ and $s_{0}^{\prime}: P_{\widetilde{B}_{2}} \rightarrow P_{\widetilde{A}_{2}}$.

Now we must compare the coefficients of the same term $\left[M_{\bullet}\right]=\left[N_{\bullet}\right]$ in (2.9) and (2.10). A quick observation yields that we must have the correspondences

$$
Q_{\widetilde{A}_{2}}=Q_{B_{1}}, \quad Q_{\widetilde{B}_{2}}=P_{B_{1}}, \quad P_{\widetilde{B}_{2}}=P_{A_{1}}, \quad P_{\widetilde{A}_{2}}=Q_{A_{1}}
$$

and

$$
f_{\widetilde{B}_{2}}=-s_{1}, \quad f_{\widetilde{A}_{2}}=s_{0}, \quad s_{1}^{\prime}=-f_{B_{1}}, \quad s_{0}^{\prime}=f_{A_{1}} .
$$

Then from (2.7) and (2.11), we have the next combined diagram

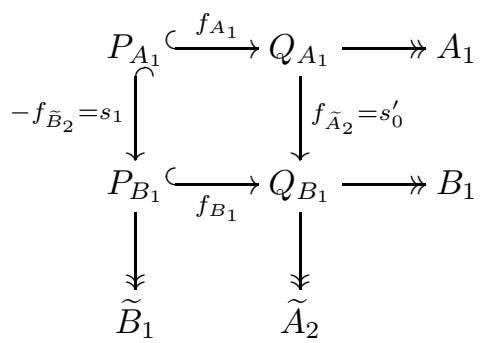

where all the columns and rows are short exact. We also have the short exact sequences

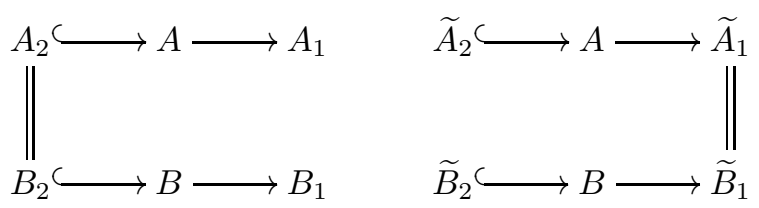

Considering the classes in the Grothendieck group $K(\mathcal{A})$, we have the relations

$$
\begin{aligned}
& \widehat{\widetilde{A}}_{1}=\widehat{A}-\widehat{\widetilde{A}}_{2}=\widehat{A}-\left(\widehat{Q}_{B_{1}}-\widehat{Q}_{A_{1}}\right) \\
= & \widehat{\widetilde{B}}_{1}=\widehat{B}-\widehat{\widetilde{B}}_{2}=\widehat{B}-\left(\widehat{P}_{B_{1}}-\widehat{P}_{A_{1}}\right)
\end{aligned}
$$

and

$$
\begin{aligned}
\widehat{A}_{2} & =\widehat{A}-\widehat{A}_{1}=\widehat{A}-\left(\widehat{Q}_{A_{1}}-\widehat{P}_{A_{1}}\right) \\
=\widehat{B}_{2} & =\widehat{B}-\widehat{B}_{1}=\widehat{B}-\left(\widehat{Q}_{B_{1}}-\widehat{P}_{B_{1}}\right) .
\end{aligned}
$$

Then we have

$$
\begin{aligned}
\widehat{A}-\widehat{B} & =\left(\widehat{Q}_{B_{1}}-\widehat{Q}_{A_{1}}\right)-\left(\widehat{P}_{B_{1}}-\widehat{P}_{A_{1}}\right) \\
& =\left(\widehat{Q}_{A_{1}}-\widehat{P}_{A_{1}}\right)-\left(\widehat{Q}_{B_{1}}-\widehat{P}_{B_{1}}\right),
\end{aligned}
$$

so that in $K(\mathcal{A})$ we have

$$
\widehat{Q}_{B_{1}}+\widehat{P}_{A_{1}}=\widehat{Q}_{A_{1}}+\widehat{P}_{B_{1}}, \quad \widehat{A}_{1}=\widehat{B}_{1}, \quad \widehat{\widetilde{A}}_{2}=\widehat{\widetilde{B}}_{2}
$$

Now we will finish the proof. By the correspondences (2.12) and (2.13), we have only to check that the coefficients

$$
\left\langle A_{1}+B_{1}, A_{2}\right\rangle+\left\langle P_{A_{1}}, A_{1}\right\rangle+\left\langle P_{B_{1}}, B_{1}\right\rangle-\left(P_{B_{1}}, A_{1}\right)-\left\langle P_{A_{1}}, Q_{B_{1}}\right\rangle+\left\langle Q_{A_{1}}, P_{B_{1}}\right\rangle
$$


in (2.9) and

$$
\left\langle\widetilde{A}_{1}, \widetilde{A}_{2}+\widetilde{B}_{2}\right\rangle+\left\langle P_{\widetilde{B}_{2}}, \widetilde{B}_{2}\right\rangle+\left\langle P_{\widetilde{A}_{2}}, \widetilde{A}_{2}\right\rangle-\left(P_{\widetilde{A}_{2}}, \widetilde{B}_{2}\right)+\left\langle Q_{\widetilde{B}_{2}}, P_{\widetilde{A}_{2}}\right\rangle-\left\langle P_{\widetilde{B}_{2}}, Q_{\widetilde{A}_{2}}\right\rangle
$$

in (2.10) coincide. But using (2.14) we have

$$
\begin{aligned}
\underline{(2.15)}= & \left\langle A_{1}+B_{1}, A\right\rangle-\left\langle A_{1}+B_{1}, A_{1}\right\rangle+\left\langle P_{A_{1}}, A_{1}\right\rangle+\left\langle P_{B_{1}}, B_{1}\right\rangle \\
& -\left\langle P_{B_{1}}, A_{1}\right\rangle-\left\langle A_{1}, P_{B_{1}}\right\rangle-\left\langle P_{A_{1}}, Q_{B_{1}}\right\rangle+\left\langle Q_{A_{1}}, P_{B_{1}}\right\rangle \\
= & 2\left\langle A_{1}, A_{2}\right\rangle+\left\langle P_{A_{1}}, A_{1}\right\rangle-\left\langle A_{1}, P_{B_{1}}\right\rangle-\left\langle P_{A_{1}}, Q_{B_{1}}\right\rangle+\left\langle Q_{A_{1}}, P_{B_{1}}\right\rangle \\
= & 2\left\langle A_{1}, A_{2}\right\rangle+\left\langle P_{A_{1}}, \widehat{A}_{1}-\widehat{Q}_{B_{1}}\right\rangle+\left\langle\widehat{Q}_{A_{1}}-\widehat{A}_{1}, P_{B_{1}}\right\rangle \\
= & 2\left\langle A_{1}, A_{2}\right\rangle+\left\langle P_{A_{1}},-\widehat{P}_{B_{1}}\right\rangle+\left\langle\widehat{P}_{A_{1}}, P_{B_{1}}\right\rangle=2\left\langle A_{1}, A_{2}\right\rangle .
\end{aligned}
$$

A similar calculation gives

$$
\text { (2.16) }=2\left\langle A_{1}, A_{2}\right\rangle \text {. }
$$

Thus the proof is completed.

\section{Concluding Remarks}

As mentioned in [1, §1.4], the work of Cramer [3] and Theorem 1.26 yield the following:

Corollary 3.1. For the hereditary abelian cateogry, the algebra $\mathcal{D H}(\mathcal{A})$ is functorial with respect to derived invariance.

Precisely speaking, let $\mathcal{A}$ and $\mathcal{B}$ be two abelian categories satisfying conditions (a)-(e). Assume that the bounded derived categories $D^{b}(\mathcal{A})$ and $D^{b}(\mathcal{B})$ of $\mathcal{A}$ and $\mathcal{B}$ are equivalent by the functor $\Phi$ :

$$
\Phi: D^{b}(\mathcal{A}) \stackrel{\sim}{\longrightarrow} D^{b}(\mathcal{B}) .
$$

Then one can construct an algebra isomorphism

$$
\Phi^{\mathcal{D H}}: \mathcal{D H}(\mathcal{A}) \stackrel{\sim}{\longrightarrow} \mathcal{D H}(\mathcal{B}),
$$

and this construction is functorial: $\left(\Phi_{1} \circ \Phi_{2}\right)^{\mathcal{D H}}=\Phi_{1}^{\mathcal{D H}} \circ \Phi_{2}^{\mathcal{D H}}$ for all equivalences $\Phi_{1}, \Phi_{2}$.

Now set $\mathcal{T}:=D^{b}(\mathcal{A})$ for some hereditary abelian category $\mathcal{A}$ satisfying (a)-(e). We also set

$$
\mathcal{D H}(\mathcal{T}):=\mathcal{D} \mathcal{H}(\mathcal{A})
$$

This algebra depends only on the triangulated cateogry $\mathcal{T}$ by the above corollary. Denote by $\operatorname{Auteq}(\mathcal{T})$ the group of autoequivalences of $\mathcal{T}$. Then, setting

$$
\operatorname{Aut}^{\mathcal{D H}}(\mathcal{T}):=\left\{\Phi^{\mathcal{D H}} \mid \Phi \in \operatorname{Auteq}(\mathcal{T})\right\}
$$

we have an embedding of groups

$$
\operatorname{Aut}^{\mathcal{D H}}(\mathcal{T}) \subset \operatorname{Aut}(\mathcal{D H}(T))
$$

Let us close this note by mentioning a non-trivial example. For an elliptic curve $C$ defined over $\mathfrak{k}$, set

$$
\mathcal{A}:=\operatorname{Coh}(C),
$$

the abelian category of coherent sheaves on $C$. This category satisfies the conditions (a)-(e). Set $\mathcal{T}:=D^{b}(\mathcal{A})$ as above.

Then by the theory of Fourier-Mukai transforms, we have a short exact sequence

$$
0 \longrightarrow \mathbb{Z} \oplus(C \times \widehat{C}) \longrightarrow \operatorname{Auteq}(\mathcal{T}) \longrightarrow \mathrm{SL}_{2}(\mathbb{Z}) \longrightarrow 0
$$

of groups. Here $\mathbb{Z} \oplus(C \times \widehat{C})$ corresponds to the subgroup of Auteq $(\mathcal{T})$ generated by the shifts $[n]$ of complexes, the pushforward $t_{a *}$ by translations on $C$ with $a \in C$, and tensor products $L \otimes(-)$ with $L \in \widehat{C}:=\operatorname{Pic}^{0}(C)$. The cokernel part $\mathrm{SL}_{2}(\mathbb{Z})$ consists of (non-trivial) Fourier-Mukai transforms $\Phi_{\mathcal{E}}:=\mathbb{R} p_{2 *}\left(\mathcal{E} \stackrel{\mathbb{L}}{\otimes} p_{1}^{*}(-)\right)$ with $\mathcal{E} \in D^{b}(C \otimes \widehat{C})$. The generators $S:=\left(\begin{array}{cc}0 & -1 \\ 1 & 0\end{array}\right)$ and $T:=\left(\begin{array}{cc}1 & -1 \\ 0 & 1\end{array}\right)$ correspond to the Fourier-Mukai transforms $\Phi_{\mathcal{E}_{0}}$ and $L \otimes(-)$, where $\mathcal{E}_{0}$ is the Poincaré bundle on $C \otimes \widehat{C}$, and $L \in \operatorname{Pic}(C)$ is a degree one line bundle. (See [8, §9.5] for the detailed explanation.)

Now one can see that the operation

$$
D H: \Phi \longmapsto \Phi^{\mathcal{D H}}
$$


yields

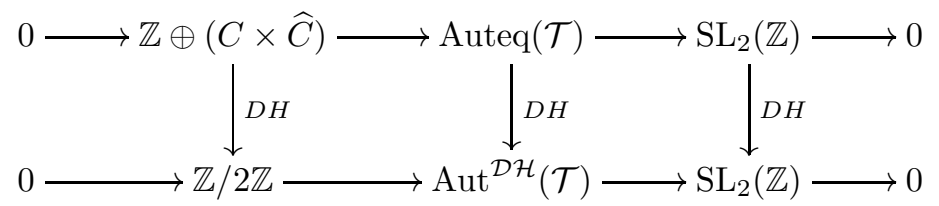

Here $\mathbb{Z} / 2 \mathbb{Z}$ corresponds to the involution $*$ of the algebra $\mathcal{D H}(\mathcal{T})$. Thus $\mathrm{SL}_{2}(\mathbb{Z})$ acts on $\mathcal{D} \mathcal{H}(\mathcal{T})$. This action is essentially the same as the $\mathrm{SL}_{2}(\mathbb{Z})$-automorphisms of the algebra appearing in $[2]$. The same algebra appeared in the works [4, [5], 6] and [12]. The $\mathrm{SL}_{2}(\mathbb{Z})$-automorphisms (precisely speaking, the counterpart in the degenerate algebra) play an important role in the argument of [13] in the context of the so-called AGT relation/conjecture.

\section{REFERENCES}

[1] Bridgeland, T., Quantum groups via Hall algebras of complexes, arXiv:1111.0745

[2] Burban, I., Schiffmann, O., On the Hall algebra of an elliptic curve, I, Duke Math. J. 161 (2012), no. 7, 1171-1231.

[3] Cramer, T., Double Hall algebras and derived equivalences, Adv. Math. 224 (2010), no. 3, 1097-1120.

[4] Feigin, B., Feigin, E., Jimbo, M., Miwa, T., Mukhin, E., Quantum continuous $\mathfrak{g l}_{\infty}$ : tensor products of Fock modules and $\mathcal{W}_{n}$-characters, Kyoto J. Math. 51 (2011), no. 2, 365-392.

[5] Feigin, B, Hashizume, K., Hoshino, A., Shiraishi, J., Yanagida, S., A commutative algebra on degenerate CP1 and Macdonald polynomials, J. Math. Phys. 50 (2009), no. 9, 095215, 42 pp.

[6] Feigin, B., Tsymbaliuk, A., Equivariant K-theory of Hilbert schemes via shuffle algebra, Kyoto J. Math. 51 (2011), no. 4 , $831-854$.

[7] Green, J., Hall algebras, hereditary algebras and quantum groups, Invent. Math. 120 (1995), no. 2, 361-377.

[8] Huybrechts, D., Fourier-Mukai transforms in algebraic geometry, Oxford Mathematical Monographs, Oxford University Press, 2006.

[9] Joseph, A., Quantum groups and their primitive ideals, Ergebnisse der Mathematik und ihrer Grenzgebiete (3), 29, Springer-Verlag, Berlin, 1995.

[10] Ringel, C., Hall algebras and quantum groups, Invent. Math. 101 (1990), no. 3, 583-591.

[11] Schiffmann, O., Lectures on Hall algebras, arXiv:0611617v2.

[12] Schiffmann, O., Vasserot, E., The elliptic Hall algebra and the equivariant $K$-theory of the Hilbert scheme of $\mathbb{A}^{2}$, preprint, arXiv:0905.2555 2 .

[13] Schiffmann, O., Vasserot, E., Cherednik algebras, $W$ algebras and the equivariant cohomology of the moduli space of instantons on $\mathbb{A}^{2}$, preprint, arXiv:1202.2756

[14] Xiao, J., Drinfeld double and Ringel-Green theory of Hall algebras, J. Algebra 190 (1997), no. 1, 100-144.

Research Institute for Mathematical Sciences, Kyoto University, Kyoto 606-8502, Japan

E-mail address: yanagida@kurims.kyoto-u.ac.jp 